\section{Granulomas pulmonares y en médula ósea: más allá de la tuberculosis. A propósito de un caso}

\author{
CAMILA PEÑA ${ }^{1}$, ANDRÉS SOTO ${ }^{2}$, PABLO VILLEGAS ${ }^{3}$
}

\section{Differential diagnosis of bone marrow and lung granulomas. Report of one case}

The differential diagnosis of pulmonary tuberculosis and lymphoma with pulmonary infiltration is very difficult, given their similar clinical characteristics. We report a 59 year old female with weight loss, fever, dyspnea and cough of several months of duration. She had a cavitated mass in lung imaging. A positive conventional PCR lead to the diagnosis of tuberculosis, but she had negative smears and cultures for Mycobacterium tuberculosis. The patient did not respond to treatment and her clinical condition worsened. A peripheral lymph node biopsy confirmed the presence of a diffuse large B cell non-Hodgkin lymphoma. Bone marrow pathology showed non caseating granulomas, again with negative microbiological tests for $M$. tuberculosis. Findings in the bone marrow were interpreted as a secondary sarcoid reaction to cancer and PCR results as a false positive. The lymphoma was treated, achieving complete remission. This case highlights the importance of the differential diagnosis between these two entities.

(Rev Med Chile 2017; 145: 657-661)

Key words: Diagnosis, Differential; Granuloma; Non-Hodgkin Lymphoma; Polymerase Chain Reaction; Tuberculosis, Pulmonary.
'Sección Hematología, Hospital del Salvador. Santiago, Chile. ${ }^{2}$ Sección Infectología, Hospital del Salvador. Santiago, Chile. ${ }^{3}$ Servicio de Anatomía Patológica, Hospital del Salvador. Santiago, Chile.

Recibido el 26 de abril de 2016, aceptado el 22 de marzo de 2017.

Correspondencia a: Dra. Camila Peña O. camipena@gmail.com
$\mathrm{T}$ lanto la tuberculosis pulmonar (TBCP) como el linfoma con infiltración pulmonar pueden presentarse con clínica de evolución crónica, incluyendo síntomas consuntivos como fiebre, baja de peso o diaforesis nocturna. También hallazgos al examen físico como adenopatías, y la presencia de lesiones tipo nódulos o masas, con o sin cavitaciones en las imágenes pulmonares. Incluso en la histología puede encontrarse granulomas en ganglios y en la médula ósea (MO) en ambas patologías. Esto hace que su diagnóstico diferencial sea, en algunas ocasiones, en extremo complejo.

Presentamos el caso de una paciente cuya presentación clínica, radiológica y de laboratorio hizo pensar inicialmente en una TBCP, pero en la que el estudio posterior confirmó un linfoma no Hodgkin difuso de células grandes B (LNH DCGB).

\section{Caso clínico}

Paciente de sexo femenino, de 59 años, hipertensa. Antecedente de TBCP en la adolescencia, tratada. Historia de 8 meses de fiebre hasta 39 ${ }^{\circ} \mathrm{C}$ y tos productiva. Fue manejado con distintos esquemas antimicrobianos, sin respuesta. Se hospitalizó inicialmente en otro centro, donde se realizó una tomografía computada (TC) de tórax que mostró opacidades masiformes en lóbulo inferior izquierdo y múltiples nódulos pulmonares bilaterales cavitadas (Figura 1). El examen para VIH fue negativo. Las baciloscopías (BK) y los cultivos de Koch de expectoración resultaron negativos. Se realizó un lavado bronquioalveolar (LBA) y biopsia, cuyo estudio histológico transbronquial reveló un proceso inflamatorio crónico granulomatoso, sin células 
neoplásicas, con tinción de Ziehl-Nielsen negativa. Cultivo corriente aerobio, de hongos, BK y cultivo de Koch resultaron negativos. Se realizó además una reacción en cadena de la polimerasa (PCR) para M. tuberculosis, con el kit comercial AMPLICOR ${ }^{\circledR}$ (Roche Diagnostics System, USA), cuyo resultado fue positivo, por lo que el cuadro fue interpretado como una probable recidiva de TBCP y se manejó con isoniazida, rifampicina y pirazinamida según norma ministerial vigente, siendo dada de alta. A pesar de esto, la paciente persistió con marcado compromiso del estado general, se mantuvo febril y con progresiva baja de peso de $20 \mathrm{~kg}$ en los siguientes 3 meses.

Dada esta mala evolución, la paciente fue hospitalizada en el Hospital del Salvador, para estudio. Se realizó nueva FBC con biopsia transbronquial, la cual mostró infiltración difusa de células grandes de aspecto linfoide. Sin embargo, la inmunohistoquímica (IHQ) no logró confirmar un linfoma. El estudio microbiológico bacteriano, fúngico y micobacteriano fue nuevamente negativo. La paciente continuó febril y con progresión de las masas pulmonares evidenciado en una nueva TC de tórax. Se realizó una nueva biopsia, esta vez quirúrgica por videotoracoscopía. La muestra histológica y la IHQ mostraron un pulmón con infiltrado de tipo linfoide reactivo. Evolucionó en 1 mes con aparición de adenopatías cervicales e inguinales de $2 \mathrm{~cm}$, no adheridas a planos profundos, no fistulizadas ni dolorosas. Se efectuó biopsia de una adenopatía inguinal, que finalmente confirmó

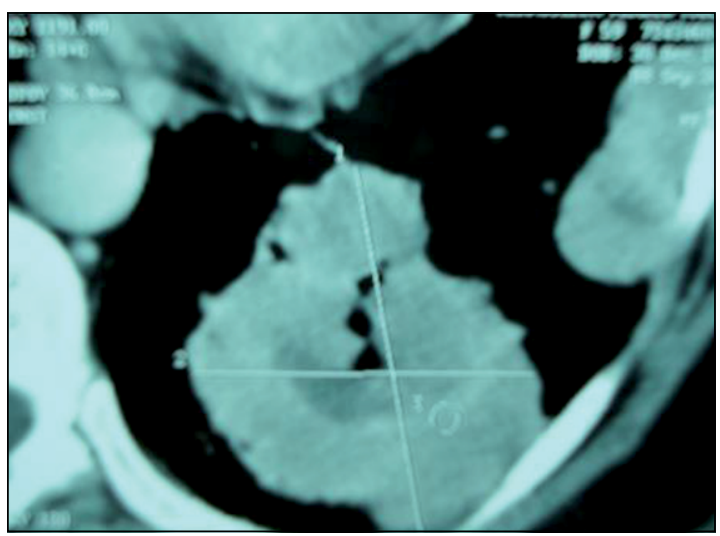

Figura 1. TC de tórax que muestra la lesión pulmonar cavitada inicial. un LNH DCGB anaplástico mediante IHQ (positivo para CD20, BCL-2, CD30). Se suspendió el tratamiento antituberculoso, ya estando en la segunda semana de la fase bisemanal.

La TC de tórax, abdomen y pelvis de etapificación evidenció múltiples adenopatías mediastínicas, axilares, retroperitoneales, infracavo-aórticas y en cadenas iliacas, la mayor de $3 \mathrm{~cm}$, además de múltiples nódulos pulmonares $(4 \mathrm{~cm})$ y hepatoesplenomegalia moderada. La biopsia de MO mostró alta celularidad, con granulomas de células gigantes multinucleadas no necrosadas (Figura 2). No se encontró proceso neoplásico linfoide ni a la microscopía ni con inmunohistoquímica y la tinción de Ziehl-Nielsen resultó negativa. Nuevamente se planteó dudas sobre el rol de una infección tuberculosa diseminada como diagnóstico concomitante. Se realizó BK, cultivo de Koch y PCRAMPLICOR ${ }^{\circledR} M$. tuberculosis en $\mathrm{MO}$, que resultaron negativos. El caso fue evaluado por un equipo multidisciplinario, y se plantea en conjunto el diagnóstico definitivo de linfoma, con compromiso pulmonar y reacción sarcoide en MO. Se planteó que la PCR positiva para $M$. tuberculosis de LBA corresponde a cepa no viable, en contexto de infección antigua. Se inició quimioterapia con rituximab, ciclofosfamida, vincristina, doxorrubicina y prednisona (R-CHOP) con muy buena respuesta. Se administraron 6 ciclos, logrando remisión completa (RC) de la enfermedad. Actualmente, la paciente se encuentra en control, manteniéndose en RC desde hace 5 años.

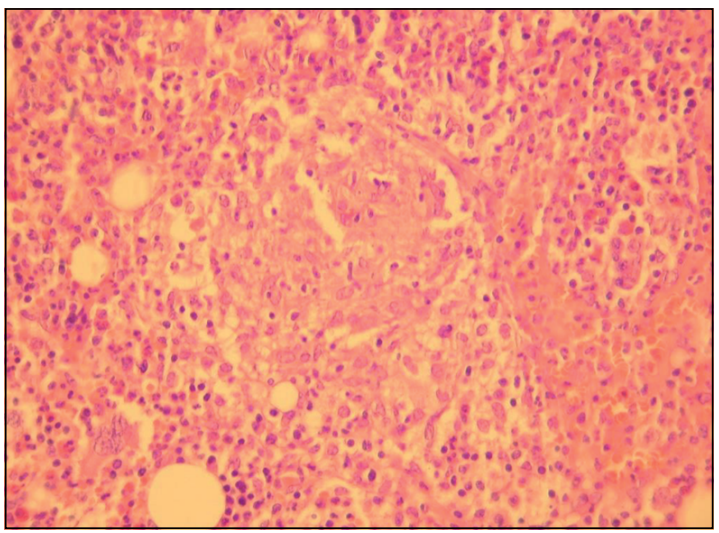

Figura 2. Granuloma en corte de biopsia de médula ósea de la paciente (Tinción Hematoxilina-eosina). 


\section{Discusión}

La TBC y el linfoma tienen varias características clínicas en común, como baja de peso, fiebre, diaforesis nocturna y poliadenopatías ${ }^{1}$.

Dentro de las lesiones pulmonares cavitadas, las etiologías más frecuentes son abscesos piógenos, tumores cavitados y las infecciones por micobacterias y hongos ${ }^{2}$. La TBCP es uno de los primeros diagnósticos a considerar, pero es importante tener en cuenta que un tumor sólido o un linfoma agresivo de rápido crecimiento puede cavitarse, necrosándose su centro por falta de vascularización.

Un hecho clave, fue el haber diagnosticado la TBCP sólo a través de la PCRAMPLICOR ${ }^{\circledR} M$. tuberculosis, sin tomar en cuenta que las múltiples BK y cultivos de Koch (expectoración y LBA) fueron negativas, resultados inhabituales en pacientes con cavitaciones pulmonares por TBCP, por lo general bacilíferos. La sola positividad de una PCR no es suficiente para el diagnóstico de $\mathrm{TBC}^{3}$, más aun considerando el antecedente obtenido retrospectivamente de una TBCP en la infancia. Existen varios estudios que demuestran que este kit comercial tiene una sensibilidad sobre el 80\% y especificidad que superan el $90-95 \%$ en muestras respiratorias ${ }^{4,5}$, pero como cualquier método de biología molecular no diferencia agentes vivos de aquellos muertos no viables. Por otro lado, hay algunos reportes que han mencionado que algunos kit comerciales actualmente menos usados, como éste, no eran capaces de diferenciar $M$. tuberculosis de otras micobacterias pertenecientes al complejo M. tuberculosis, como M. bovis ${ }^{6}$. Cabe destacar que el gold standard para el diagnóstico de la TBC sigue siendo el cultivo de Koch, por su alta sensibilidad ${ }^{4-6}$. Todos los cultivos realizados en la paciente resultaron negativos. Al momento de la presentación del caso, no había disponibilidad en nuestro medio de PCR MTB/RIF para $M$. tuberculosis (GeneXpert $\left.{ }^{\circledR}\right)$, técnica que ha logrado mejorar la sensibilidad y la especificidad diagnóstica de esta micobacteria, tanto en muestras respiratorias como extrapulmonares, destacando además su rapidez y la capacidad de detectar el gen rpoB, que codifica la resistencia a rifampicina ${ }^{7,8}$. Por estas ventajas comparativas, este es el examen recomendado en la actualidad por la OMS para el diagnóstico de la TBC.

Por otro lado, una vez se creyó certificado el diagnóstico de $\mathrm{LNH}$, la biopsia de $\mathrm{MO}$ informó granulomas, por lo que nuevamente se planteó una TBC. La identificación de granulomas epitelioides no caseificantes semejantes a los de la sarcoidosis, es conocida como reacción sarcoide (RS). Se desconoce su causa. Se ha postulado como mecanismo patogénico la presencia de antígenos derivados de un tumor necrosado que pudieran causar reacciones de hipersensibilidad mediada por células $\mathrm{T}$ helper, es decir, probablemente se deben a factores antigénicos de las células tumorales que generan una respuesta inmune de hipersensibilidad de tipo IV. Otra teoría es que factores quimiotácticos derivados de un tumor estimulan la migración de monocitos y los activan para formar histiocitos epitelioides o células gigantes ${ }^{8}$. Por lo tanto, se cree que esta reacción es una manifestación defensiva del organismo para prevenir la diseminación del tumor. Esto podría ser un marcador de respuesta antitumoral mediada inmunológicamente y la demostración de una mejor evolución y pronóstico ${ }^{9-11}$.

Las localizaciones más frecuentes de la RS, además de ganglios linfáticos, incluyen piel, huesos, hígado, bazo y pulmón. Esto podría explicar que la primera muestra histológica tomada del LBA mostró lesiones granulomatosas y no linfoma. Varios tipos de tumores pueden inducir este patrón morfológico. Brincker la describió en 4,4\% de pacientes con carcinoma, 13,8\% en linfoma de Hodgkin (LH), y entre 3,6\% y 7,3\% en $\mathrm{LNH}^{12}$.

Los granulomas (o RS) en MO son infrecuentes. Un estudio reciente revisó 5.217 biopsias de MO desde el 2001 al 2007, y se encontró sólo 20 con granulomas $(0,38 \%)$. Trece $(65 \%)$ fueron diagnosticados como TBC. Cuatro tuvieron como diagnóstico tumores hematológicos y 2 hepatitis viral $^{13}$. Esta baja incidencia se confirma en otros estudios, que muestran granulomas en $\mathrm{MO}$ en 0,3 a $2,2 \%{ }^{13-19}$. Se ha implicado varias enfermedades asociadas, y las neoplasias dan cuenta de aproximadamente el 20-25\% de los casos. El más común es el LH seguido de algunos carcinomas. Los LNH (como nuestro caso) son menos frecuentes, con el 2,7\% de los $\operatorname{casos}^{18}$. Los tipos de LNH más comúnmente asociados a granulomas de $\mathrm{MO}$ son linfoma folicular, linfoma linfocítico de células pequeñas, linfomas de células grandes $\mathrm{B}$ y algunos de células $\mathrm{T}^{19}$.

Las infecciones son otra causa importante. $\mathrm{La}$ TBC cuando es diseminada, puede manifestarse 
con granulomas en MO entre 6-48\% de los casos, aún más en pacientes inmunocomprometidos ${ }^{20-21}$. Al contrario de lo esperable en estos casos, se observan granulomas caseificantes en sólo $29 \%$ de los $\operatorname{casos}^{21}$. Otras causas descritas son la brucelosis, fiebre tifoidea, leishmaniasis, infección por citomegalovirus, virus Epstein Barr, e infecciones fúngicas diseminadas ${ }^{22}$.

Finalmente, se debe considerar que siempre existirá la posibilidad de presentar las dos enfermedades concomitantemente ${ }^{23}$, más aún en pacientes inmunocomprometidos como trasplantados o pacientes con VIH, lo que hace más complejo su diagnóstico.

\section{Conclusión}

Este caso refleja lo difícil que es a veces diferenciar entidades que parecerían de fácil diagnóstico, como es la TBCP y el linfoma. Es importante tener presente la similitud de ambas presentaciones clínicas y, por lo tanto, que se debe hacer un estudio acabado para llegar al diagnóstico.

\section{Referencias}

1. Petousi N, Mathew J, Thomas EC. A patient presenting with generalized lympadenopathy-sarcoidosis, lymphoma or tuberculosis? BMJ Case Rep 2012; 2012.

2. Abi Khalil S, Gourdier AL, Aoun N, Nedelcu C, El Rai S, Moubarak E, et al. Cystic and cavitary lesions of the lung: imaging characteristics and differential diagnosis. J Radiol 2010; 91 (4): 465-73.

3. Restrepo B. Nuevas herramientas para la detección de la tuberculosis latente. Biomédica 2004; 24 (Supl.): 202-11.

4. Bloemberg GV, Voit A, Ritter C, Deggim V, Böttger EC. Evaluation of Cobas TaqMan MTB for direct detection of the Mycobacterium tuberculosis complex in comparison with Cobas Amplicor MTB. J Clin Microbiol 2013; 51 (7): 2112-7.

5. Selman C, Poggi H, Román JC, García P, Lagos M. Análisis retrospectivo del rendimiento de Amplicor-PCR ${ }^{\circledR}$ para la detección de Mycobacterium tuberculosis en muestras respiratorias y no respiratorias con baciloscopia negativa. Rev Chilena Infectol 2009; 26 (6): 495-8.

6. Noordhoek GT, Kolk AH, Bjune G, Catty D, Dale JW, Fine PE, et al. Sensitivity and Specifity of PCR for Detection of Mycobacterium tuberculosis: a Blind Comparison
Study Among Seven Laboratories. J Clin Microbiol 1994; 32 (2): 277-84.

7. Denkinger CM, Schumacher SG, Boehme CC, Dendukuri N, Pai M, Steingart KR. Xpert MTB/RIF assay for the diagnosis of extrapulmonary tuberculosis: a systematic review and meta-analysis. Eur Respir J 2014; 44 (2): 43546.

8. Boehme CG, Nabeta P, Hillemann D, Nicol MP, Shenai S, Krapp F, et al. Rapid molecular detection of tuberculosis and rifampin resistance. N Engl J Med 2010; 363 : 1005-15.

9. Kurata A, Terado Y, Schulz A, Fujioka Y, Franke FE. Inflammatory cells in the formation of tumor-related sarcoid reactions. Human Pathol 2005; 36: 546-54.

10. Braylan RC, Long JC, Jaffe ES, Greco FA, Orr SL, Berard CW. Malignant lymphoma obscured by concomitant extensive epithelioid granulomas. Cancer 1997; 39: 1146-55.

11. Hollingsworth HC, Longo DL, Jaffe ES. Small noncleaved cell lymphoma associated with florid epithelioid granulomatous response. A clinicopathologic study of seven patients. Am J Surg Pathol 1993; 17: 51-9.

12. Brincker H. Sarcoid reactions in malignant tumours. Cancer Treat Rev 1986; 13: 147-56.

13. Feng J, Zhang H, Zhong DR. A clinical analysis of 20 cases with bone marrow granulomas, Zhonghua Nei Ke Za Zhi 2009; 48 (6): 485-7.

14. Eid A, Carion W, Nystrom JS. Differential Diagnoses of Bone Marrow Granuloma. West J Med 1996; 164: 510-5.

15. Bhargava V, Farhi DC. Bone marrow granulomas: Clinicopathologic findings in 72 cases and review of the literature. Hematol Pathol 1988; 2: 43-50.

16. Pease G. Granulomatous lesion in the bone marrow. Blood 1956; 11: 720-34.

17. Brackers de Hugo L, French M, Broussolle C, Sève P. Granulomatous lesions in bone marrow: clinicopathologic findings and significance in a study of 48 cases. Eur J Intern Med 2013; 24 (5): 468-73.

18. Choe JK, Hyun BH, Salazar GH, Ashton JK, Sung CY. Epithelioid granulomas of the bone marrow in non-Hodgkin's lymphoproliferative malignancies. Am J Clin Pathol 1983; 80: 19-24.

19. Dunphy CH, Panella MJ, Grosso LE. Low-Grade B-Cell Lymphoma and Concomitant Extensive Sarcoidlike Granulomas a Case Report and Review of the Literature, Arch Pathol Lab Med 2000; 124 (1): 152-6.

20. Roelandt PR, Dendooven M, De Groef K, Van Couter A. Hidden tuberculosis revealed by bone marrow biopsy as cause of fever of unknown origin, Acta Clin Belg 2006; 61 (6): 363-6.

21. Escobedo-Jaimes L, Cicero-Sabido R, Criales-Cortez JL, 
TBC y linfoma: dos grandes diagnósticos diferenciales - C. Peña et al

Ramírez E, Romero M, Rivero V, et al. Evaluation of the polymerase chain reaction in the diagnosis of miliary tuberculosis in bone marrow smear. Int J Tuberc Lung Dis 2003; 7: 580-6.

22. García P, Yrivarren JI, Argumans C, Crosby E, Carrillo C, Gotuzzo E. Evaluation of the bone marrow in pa- tients with brucellosis-Clinicopathological Correlation, Enferm Infecc Microbiol Clin 1990; 8: 19-24.

23. Fanourgiakis P, Mylona E, Androulakis II, Eftychiou C, Vryonis E, Georgala A, et al. Non-Hodgkin's lymphoma and tuberculosis coexistence in the same organs: a report of two cases. Postgrad Med J 2008; 84 (991): 276-7.

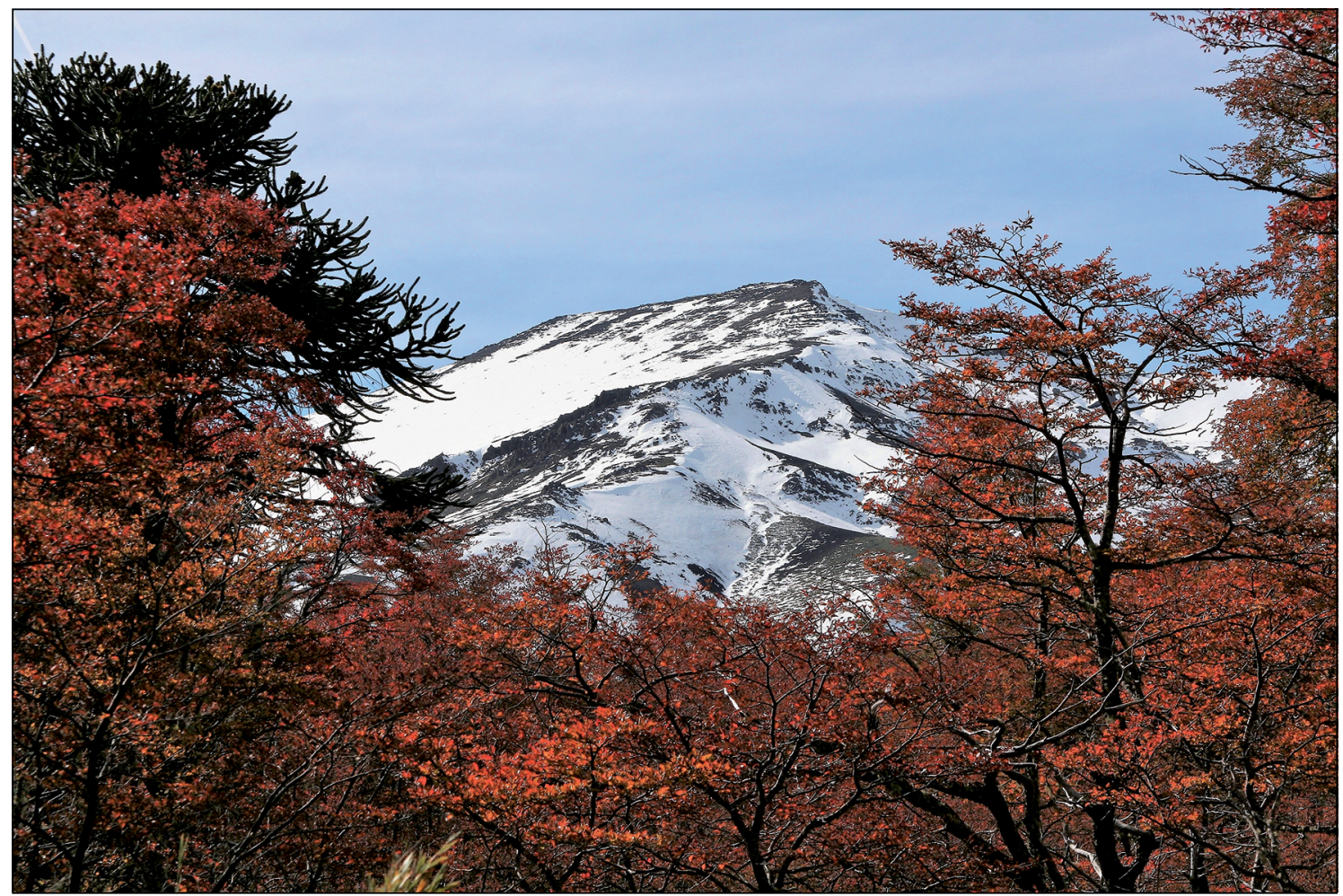

Otoño en Corralco. Dr. Jorge Sapunar Zenteno 